\title{
Spectral cleaning and output modal transformations in WGM microresonators
}

\author{
Mohd Narizee Mohd NasiR, G. Senthil Murugan, and Michalis N. Zervas*
}

Optoelectronics Research Centre, University of Southampton, Southampton, SO17 1BJ, United Kingdom.

*Corresponding author: mnz@soton.ac.uk

Received XX Month XXXX; revised XX Month, XXXX; accepted XX Month XXXX; posted XX Month XXXX (Doc. ID XXXXX); published XX Month XXXX

\begin{abstract}
A systematic study on the effects of microtaper fiber diameters on the spectral characteristics of a whisperinggallery-mode (WGM) micro-bottle resonator (MBR) is presented. Progressively cleaner and simpler spectra of the MBR were observed when the utilized microtaper fiber waist diameter $\left(D_{t}\right)$ was increased from $2 \mu \mathrm{m}$ to $10 \mu \mathrm{m}$. The maximum transmission depth at resonance varies with different microtaper fiber utilized from $\sim 20 \mathrm{~dB}\left(D_{t}=2 \mu \mathrm{m}\right)$ to $\sim 4 \mathrm{~dB}\left(D_{t}=10 \mu \mathrm{m}\right)$. The loaded $Q$-factors were observed to be unaffected by the increase of $D_{t}$ with values of $>10^{6}$ being measured in all cases. Mode transformation of MBR was also experimentally investigated and compared to a microdisc FDTD simulation by studying near-field images of the output beam on the waist of the microtaper fibers. For the first time, experimental observation of mode transformation from LP ${ }_{01}$ to $L P_{11}$ across scanned WGM resonances is being reported. (c) 2016 Optical Society of America
\end{abstract}

OCIS codes: (130.3990) Micro-optical devices; (140.3948) Microcavity devices; (140.4780) Optical resonators.

http://dx.doi.org/10.1364/AO.99.099999

\section{Introduction}

Optical microresonators (MRs), supporting whispering gallery modes (WGMs), have created much interest in the recent years for their great potential towards miniaturization and applications in advanced optical micro-systems [1, 2]. Various microresonator geometries such as microsphere [3], microtoroid [4] and microdisc [5] are capable of storing light with high $Q$-factors and in small mode volumes. This is accomplished through the process of total internal reflection between the guiding and surrounding medium of the microcavity and the formation of WGMs. Since these types of resonators primarily confine light at their respective equatorial planes, they are generally considered as a 2-D resonator with the spectral properties being defined primarily by their diameters.

Study of optical MRs supporting WGMs has also been extended to include cylindrical structures, such as optical fibers, or MRs formed on fibers for their distinctive manner in confining light as well as for easy handling and incorporation in practical applications [6-8]. Among optical MRs fabricated on optical fibers, micro-bottle resonators (MBRs) have also been given increased attention due to their unique manner of supporting WGMs. Through the combination of WG-ring and WGbouncing ball principles, MBRs are able to support true 3-D WGM light confinement [9]. MBR shape approximates accurately a harmonicoscillator profile with its WGM numbers being defined by $m$ (azimuthal), $p$ (radial) and $q$ (axial) order $[10,11]$. The two distinctive MBR turning points correspond to regions of strong field enhancement and define the area of WGM modal confinement [9]. The presence of distinctive turning points in MBRs can be used for efficient adding/dropping functionality [12]. In contrast with spherical MRs, MBRs show highly non-degenerate resonances and very rich and complex transmission spectra [10]. This is due to the fact that as the radius of MBRs varies along the length, multiple overlapping effective cavities give rise to dense superimposed resonance spectra. It has been shown that variation of radius even in the nano-scale is sufficient for MBRs to form and trap the light close to their surface [13].

The dense WGM spectral features of MBRs are advantageous for studies where large number of resonance modes are preferred; such as in cavity quantum electro-dynamics where multiple resonances are required to be simultaneously present on a linked quantum network [1]. This however would be a serious limitation in applications, such as sensing, where monitoring of specific WGM resonance is required over a broad wavelength range. It is then necessary to have MBRs with simpler WGM spectra that are easily distinguishable and traceable over a broad spectral range. Previously, two techniques for cleaning and simplifying the complex and dense spectral features of MBRs have been demonstrated by preferentially attenuating subset of the supported WGMs. The first technique showed that efficient modal filtering can be achieved by placing high-index micro-droplets as diffractive loss elements on MBRs [11]. It was also observed that even after the spectral "clean-up", narrow spectral features would still consist of superposition of a few overlapping modes. A drawback of this method is that it is very hard to control and accurately position the micro-droplets in order to attenuate certain modes while ensuring the desired ones have survived. The second technique applied direct writing of micro-groove scars on the MBR surface with focused ion beam milling. In this case, the scars introduced to the resonator surface would scatter-out the majority of WGMs and reduce the surviving mode resonances significantly [14]. With accessible beam size of $<50 \mathrm{~nm}$, high precision micro-groove shapes and designs can allow the user to accurately perform mode 
filtering and selection on MBRs. Nevertheless, while this particular technique is highly accurate, it involves expensive equipment and a number of additional fabrication steps. In this paper, we study systematically the effect of the microtaper excitation fiber diameter $\left(D_{t}\right)$ on the MBR transmission spectrum characteristics, and propose it as an alternative very simple means of cleaning-up MBR transmission spectra. Progressively cleaner and simpler spectra were obtained when $D_{t}$ was increased from $2 \mu \mathrm{m}$ to $10 \mu \mathrm{m}$. In all cases, the measured $Q$-factor values of the MBR resonances reached $>10^{6}$. Mode transformation as a result of light coupling from MBR back to the microtaper fiber is also reported. It was observed that conversion of the $\mathrm{LP}_{01}$ microtaper fiber mode into $\mathrm{LP}_{11}$ takes place in a number of strong transmission resonances in the case of $2 \mu \mathrm{m}$ taper waist diameters. Despite the much larger modality, modal transformation was observed to be not as frequent in the case of microtaper with $8 \mu \mathrm{m}$ waist diameter. In section 2 , we discuss the experimental spectral evolution of the MBR transmission spectra with microtaper diameter and compare it with theoretical modelling results showing a very good agreement. In section 3, we present experimental evidence of modal transformation in the microtaper waist, associated with strong resonances, and compare it with numerical simulations of planar waveguide/disc-resonator systems in section 4 .

\section{Micro-bottle resonator excitation source and spectral clean-up}

The MBR utilized in the experiment was fabricated by "soften-andcompress" technique demonstrated previously [10]. As shown in Fig. 1, the microresonator attain neck-to-neck distance $L_{b}=355 \mu \mathrm{m}$, bottle diameter $D_{b}=175 \mu \mathrm{m}$ and stem diameter $D_{s}=125 \mu \mathrm{m}$. One of the important parts in MRs study is the coupling of light into and out of the microcavity. Several methods relying on energy exchange between WGMs and the evanescent part of guided modes or total internal reflection has been developed to efficiently couple light into the cavity of optical resonators. One of the earliest methods was based on prism coupling which relies on total internal reflection $[15,16]$. This method was later compacted with the utilization of angle polished optical fibers instead of prism [17]. Much recently, coupling light into WGM cavity using a fused-tapered fiber tip has also been reported [18]. To date, the best method for coupling into a WGM microcavity with high efficiency is with the use of optical fiber microtapers. It is possible to couple more than $90 \%$ of the input light into the cavity of a high- $Q$ fused silica resonator [19]. In the case of high-index barium titanate glass microspheres, it was reported that the required microtaper diameter $\left(D_{t}\right)$ to observe strong transmission resonances is $\leq 2.5 \mu \mathrm{m}[20]$.

It is possible to control the excitation of MBR WGMs by varying the microtaper fiber waist diameters. For this study, five optical fibers tapered down adiabatically with $D_{t}$ of $2,4,6,8$ and $10 \mu$ m were utilized to couple light into the cavity of the fabricated MBR. The microtapers were fabricated from standard SMF28 fibres using computercontrolled, in-house tapering facilities. With the incorporation of a three-axis micro-positioning stage, the microtaper fibers were arranged to be in contact with and at right angles to the MBR axis. Although this technique overloads the cavity and reduces the resonator's overall $Q$ -

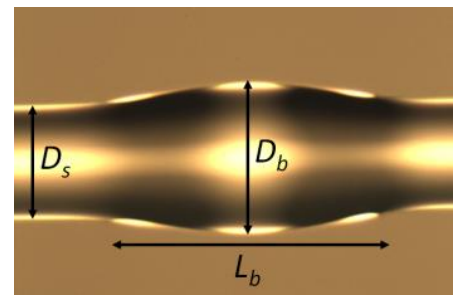

Fig. 1 Fabricated MBR of $L_{b}=355 \mu \mathrm{m}, D_{b}=175 \mu \mathrm{m}$ and $D_{s}=125 \mu \mathrm{m}$.

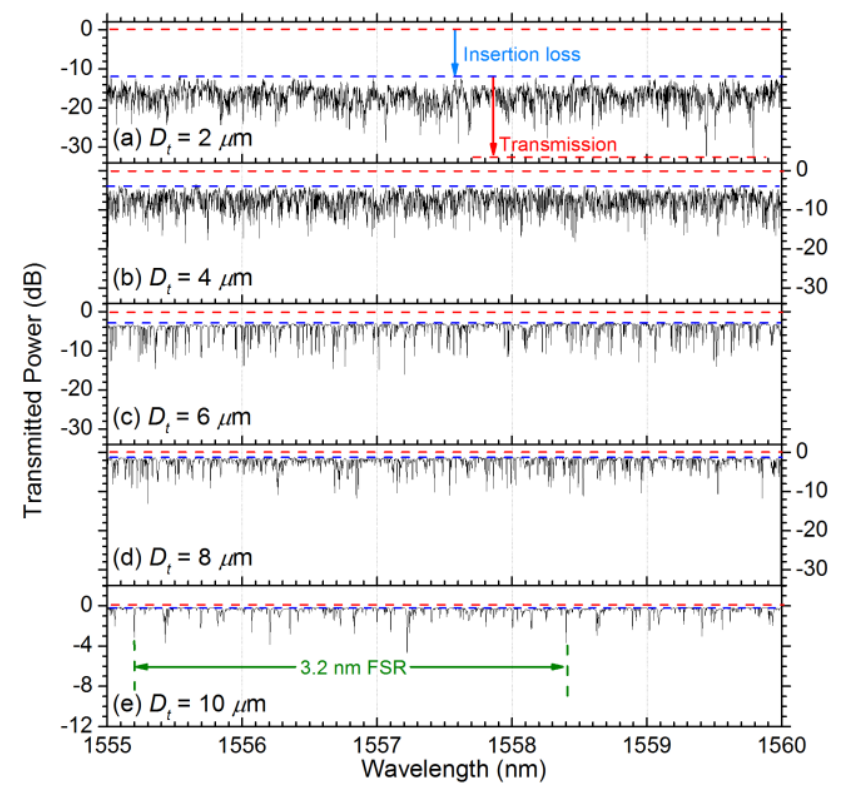

Fig. 2 Excited MBR WGM transmission spectra coupled at the center with microtapers of different waist diameter $D_{t}$.

factor value, it forms a robust and stable optical arrangement suitable for use in sensor and other practical applications. The narrow linewidth $(\sim 100 \mathrm{kHz})$ output from a tunable laser source (TLS) was launched into one end of the microtaper fiber with the other end connected to an InGaAs photo-detector for transmitted power monitoring.

Fig. 2 shows the transmission spectra of the microtaper fiber-coupled MBR when excited atcenter position with $D_{t}$ of $2,4,6,8$ and $10 \mu \mathrm{m}$. With the smallest taper diameter $D_{t}=2 \mu \mathrm{m}$, a dense transmission spectrum with strong excitation of WGMs is observed. As the taper diameter increases to $10 \mu \mathrm{m}$ the transmission spectrum becomes progressively cleaner and the maximum transmission dip decreases. In addition, strong and dense transmission spectra are accompanied by sizable insertion loss, predominantly due to excessive excitation of free-space radiation modes and a large number of partially overlapping and overcoupled WGMs (as a result of the taper being in direct contact with the MBR). The results are summarized in Fig. 3, where a decrease of the transmission dip from $\sim 20 \mathrm{~dB}$ to $\sim 4 \mathrm{~dB}$ and insertion loss from $\sim 12 \mathrm{~dB}$ to $\sim 0 \mathrm{~dB}$ is measured as the taper diameter increases from $2 \mu \mathrm{m}$ to 10 $\mu \mathrm{m}$. The observed reduction in the density of transmission resonances, i.e. spectrum clean-up, and the decrease in transmission dip with the increase of the taper diameter is a result of the variation of the coupling strength between the propagating microtaper mode and the excited WGMs. In order to quantify the coupling effects, we have modified the theoretical analysis developed for microsphere excitation by

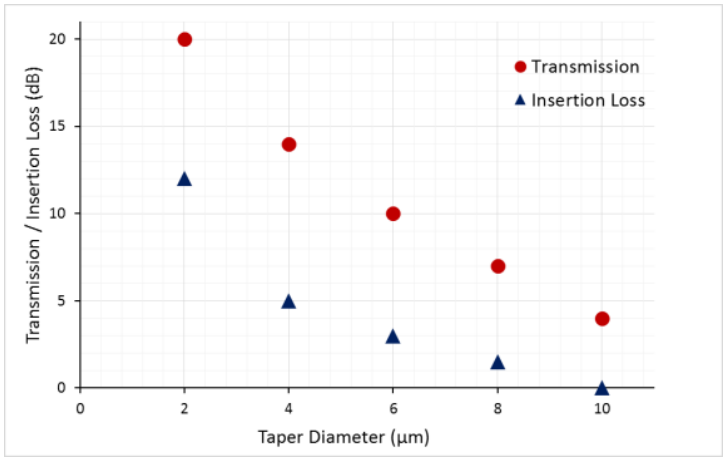

Fig. 3 Summary of MBR transmission and insertion loss vs. taper $D_{t}$. 


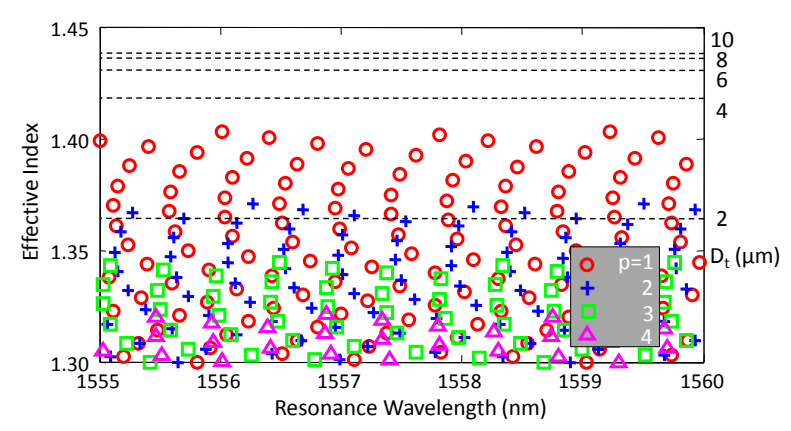

Fig. 4 Effective index of the microtaper $\mathrm{LP}_{01}$ mode $\left(D_{\mathrm{t}}=2,4,6,8\right.$, \& $10 \mu \mathrm{m})$ and MBR WGMs of different radial mode orders $(p=1,2$, $3,4)$, as a function of the resonant wavelength.

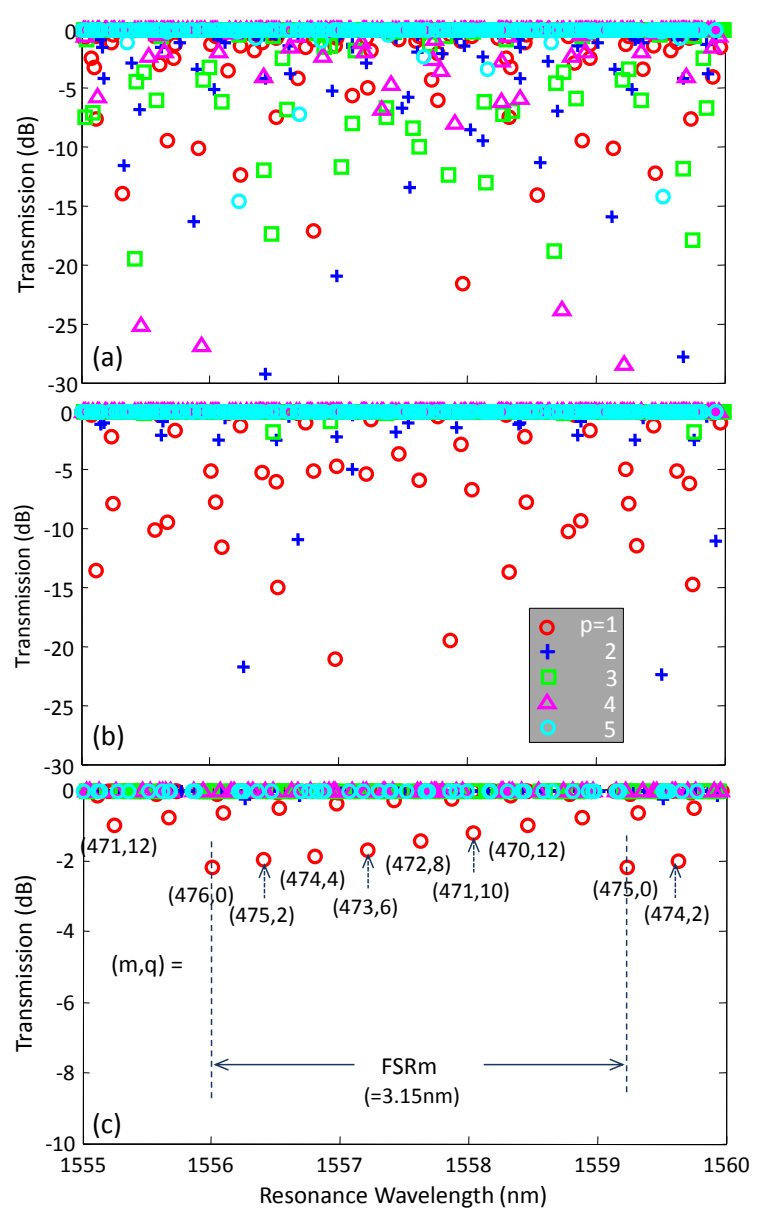

Fig. 5 WGM transmission minima vs. corresponding resonance wavelength, for different radial mode orders $(p=1,2,3,4,5)$, for microtaper diameter $D_{t}$ of (a) $2 \mu \mathrm{m}$, (b) $4 \mu \mathrm{m}$ and (c) $10 \mu \mathrm{m}$. ( $\mathrm{m}, q$ are the WGM azimuthal and axial mode numbers, respectively)

microtapers [21,22]. In Ref. [21] a quasi-parabolic profile is considered to approximate the microsphere shape, and Hermite-Gauss functions are used to describe the axial (or polar) field distributions around the coupling point. These approximations are exact expressions for the shape and axial field distributions in quasi-parabolic profile MBRs and therefore the obtained coupling constants expressions are applicable to MBRs with higher accuracy. The required radial field distributions and MBR eigenfrequencies are given in terms of $(m, q, p)$ in Ref. [11]. Details of the model will be presented elsewhere [23].
Fig. 4 shows the effective index of the microtaper $\mathrm{LP}_{01}$ mode and MBR WGMs of different radial mode orders $(p=1,2,3,4)$, as a function of the resonant wavelength. Effective indices for the taper $\mathrm{LP}_{01}$ mode and the WGMs are defined as $\beta_{\mathrm{LP} 01} / k_{0}$ and $m / R_{\mathrm{b}}$, respectively, where $\beta_{\mathrm{LP} 01}$ is propagation constant of the $\mathrm{LP}_{01}$ taper mode, $k_{0}$ is the vacuum wavenumber, $m$ is the WGM azimuthal mode number and $R_{\mathrm{b}}$ is the MBR radius. It is shown that for $D_{t}=2 \mu \mathrm{m}$ the effective index of the taper mode lies well within the WGM effective-index range and it is phasematched with a large number of WGMs. It is, therefore, expected to strongly excite a large number of WGMs supported by the MBR. As $D_{t}$ increases above the $4 \mu \mathrm{m}$ level the taper mode gets progressively more detuned from the MBR WGMs.

The impact of the $\mathrm{LP} 01$ microtaper mode effective indexvariations are shown in Fig. 5, where the transmission minima are plotted as a function of the corresponding WGM resonance wavelength. The transmission minima are calculated using a simplified coupling approach, appropriate for generalized ring-resonators [22-24]. Fig. 5 (a) and (b) show that for $D_{t}=2 \mu \mathrm{m}$ and $4 \mu \mathrm{m}$, respectively, the transmission spectra are dense due to strong excitation of many different radial order WGMs. For $D_{t}=10 \mu \mathrm{m}$ (Fig. 5 (c)), however, only the fundamental radial order $(p=1)$ WGMs are primarily excited and the calculated transmission spectrum is simplified substantially. In this case, the large phase mismatch between the microtaper LP 01 mode and the MBR WGMs (see Fig. 4), and the decreased evanescent mode field overlaps result in smaller overall coupling constantand excitation strength and, therefore, reduced transmission dips. In Fig. 5 (c) the calculated WGM azimuthal and axial mode numbers are also shown. As expected, for a microtaper placed in the MBR center, the strongest excitation corresponds to the fundamental WGM characterized by axial mode number $q=0$. In this case, the calculated free spectral range (FSR) for modes with the same $(p, q)$ mode numbers and adjacent azimuthal numbers, i.e. $(m, q, p)=$ $(476,0,1)$ and $(m, q, p)=(475,0,1)$, is $3.15 \mathrm{~nm}$, in close agreement with the experimentally obtained one (FSRexp $\approx 3.2 \mathrm{~nm}$ - see Fig. 2 (e)). We should also point out that WGMs with larger axial mode numbers show progressively smaller transmission dips, as a result of their larger axial spreading and smaller evanescent overlap with the microtaper $\mathrm{LP}_{01}$ mode. In addition, because of spatial mode symmetry, in the case of central excitation only the WGM modes with odd number of axial intensity maxima (i.e. even axial mode number $q$ ) are excited. In practice though, residual non-symmetries can result in excitation of both even and odd axial order WGMs, as it has been experimentally observed with offset excitation of slightly deformed microspheres [25]. This effect will be much more pronounced with the smaller diameter microtapers.

Utilization of microtapers with $D_{t}=10 \mu \mathrm{m}$ generated readily resolved single resonance dips which are easily distinguishable along with clearly identifiable FSR. These transmission dips can be beneficial as indication markers through any wavelength shifts in case MBRs are to be used in sensing applications [26]. In addition, we have noticed that larger microtaper fiber waist diameter also results in a much more robust device showing negligible aging and deterioration towards environmental effects, such as humidity. Conversely, microtaper fibers with $2 \mu \mathrm{m}$ waist diameters deteriorate quickly resulting in large surface scattering and transmission power drop. Comparison of Fig. 2 (a) and (e) show that the use of microtaper fiber with $D_{t}=10 \mu \mathrm{m}$ results in $>10$ $\mathrm{dB}$ throughput power increase. Fig. 6 shows specific measured resonant features of the MBR excited at center position with the five different microtaper fibers of different $D_{t}$. The wavelength span is varied in order to best demonstrate the multiple overlapping resonance fitting and the observed dominant resonance $Q$-factor (approximated by $\lambda / \Delta \lambda)$. The cumulative fitted and experimental curves are almost indistinguishable. As can be seen from the Lorentzian fitting, utilizing microtapers with $D_{t} \leq 6 \mu \mathrm{m}$ results in transmission features composed 


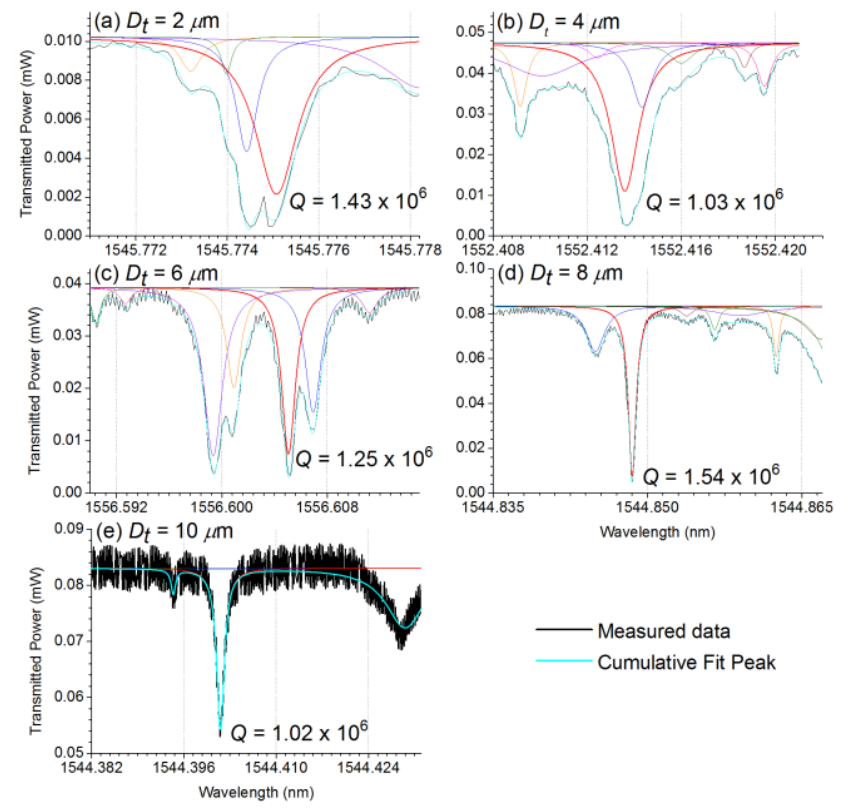

Fig. 6 Lorentzian fitting and $Q$-factor values of MBR WGMs excited with microtaper fiber of various $D_{t}$.

of a large number of overlapping WGM resonances. This is a direct consequence of the multiple radial-order WGM excitation discussed with reference to Fig. 5. When using microtapers with $D_{t} \geq 8 \mu \mathrm{m}$ the transmission features comprise predominantly single WGM resonances. As discussed above, our modelling attributes these resonances to radial order $p=1$ WGMs. This could be of great advantage for applications where monitoring single WGM dip resonances is required over a broad wavelength range. While utilizing smaller microtaper fiber waist diameters provide increased coupling efficiency and stronger resonances, this is achieved at the expense of multiple higher-radial-order MBR mode excitation. However, it should be stressed that in all cases, the measured maximum $Q$-factor of the dominant resonance was $>10^{6}$ for all microtaper waist diameters.

\section{Modal transformation of microtaper fiber by micro- bottle resonator}

Microtaper fibers utilized for exciting the MBR under study were fabricated to have an adiabatic transition from full SMF 9/125 $\mu \mathrm{m}$ core/cladding diameter to the desired diameter with $6.0 \mathrm{~mm}$ uniformwaist length. For an adiabatic microtaper fiber transition, high efficiency excitation of $\mathrm{LP}_{01}$ fundamental fiber mode reaching $99.5 \%$ could be accomplished [27]. Although the microtaper fiber supports a number of modes along its waist (where the original core diminishes and light is being guided by the waist itself), uniform-waist microtaper fibers with adiabatic transition would still maintain the high $\mathrm{LP}_{01}$ mode excitation. Placing an optical microresonator in close proximity to the microtaper fiber waist causes evanescent coupling. Two conditions must be met in order for energy transfer between the microtaper fiber to the WGM to occur. The first is the wavelength of the $\mathrm{LP}_{01}$ microtaper mode must be matched to a resonance wavelength, corresponding to a WGM of the microresonator. The second requires the coupling constant to match the microresonator WGM round trip losses for critical coupling and maximum power exchange. However, as we have seen, in the case of MBR more than one WGM can be excited simultaneously, albeit with different strengths. In a similar way, the energy back-transfer from an excited microresonator WGM is possible to take place into many different microtaper fiber modes. Even the $2 \mu \mathrm{m}$ waist microtaper

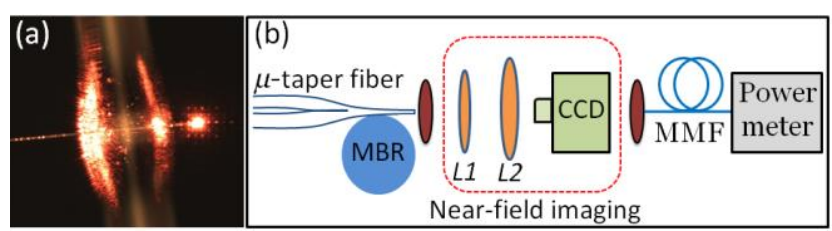

Fig. 7 (a) MBR light scattering with microtaper fiber to a He-Ne laser. (b) Schematic of microtaper fiber near-field imaging experiment.

supports $\sim 5 \mathrm{LP}$ modes at $1550 \mathrm{~nm}$. In such case, the input LP 01 mode is expected to be partially coupled into higher order waist modes after the MBR. In other words, the process of evanescently coupling into a microresonator can potentially transform the launched $\mathrm{LP}_{01}$ microtaper mode. In this section, we study experimentally the mode transformation at the output of the waist of a truncated microtaper by monitoring the near-field images of the transmitted power, as we scan across WGM resonances. The near-field images were studied across WGMs with high transmission dips as they more likely to show energy back-transfer to the microtaper waist higher-order modes.

Investigation of modal transformation from an excited MBR back to the microtaper fiber was performed with the utilization of microtaper fibers of $D_{t}=2$ and $8 \mu \mathrm{m}$. The microtaper fibers were cleaved at their respective minimum waist-regions and placed on top of the MBR as close as possible to their center, in order to minimize effects of vibration. Fig. 7 (a) shows light scattering by the MBR with cleaved microtaper fiber of $D_{t}=8 \mu \mathrm{m}$ when its input end was connected to a He-Ne laser source. Schematic of the experimental setup for scanning the transmission spectra through the terminated microtaper fibers and capturing the output beam near-field images is illustrated in Fig. 7 (b). For accurate near-field images across a WGM resonance, the output transmitted spectra were first scanned without the near-field imaging optical components (dashed-red box) set in the system. The input end of the microtaper fiber was connected to a broadband CW-TLS and an objective lens with high NA (0.65) was aligned close to the microtaper fiber cleaved point in order to collect the output light and collimate it through free-space. The light beam was then focused down by another high NA lens into a multi-mode fiber (MMF) and measured via an InGaAs optical power meter. Once the spectra of the excited MBR through the terminated microtaper fiber were acquired, optical components for capturing the output beam near-field images were placed in the system. For clear observation of modal transformations through the microtaper fibers, the collimated output beam was magnified 10X with the incorporation of two bi-convex lenses ( $L 1$ and $L 2$ ) before being directed into a CCD camera for image recording. In order to prevent image saturation on the CCD detector due to the high power going through the microtaper fiber, neutral density filters were placed in the lens barrel of the CCD camera.

Fig. 8 illustrates the observed MBR modal transformation back into the terminated microtaper fiber of one specific resonance using the finely tuned wavelength laser source with microtaper of $D_{t}=2 \mu \mathrm{m}$ across the resonance centered at the wavelength of $1557.131 \mathrm{~nm}$. Offresonance (i.e. between 1557.110 - $1557.120 \mathrm{~nm}$ - images (a) \& (b)), only the fundamental LP $\mathrm{L}_{01}$ mode was observed. Around $1557.125 \mathrm{~nm}$, there was a group of simultaneously excited WGMs. The near field image at this point shows the output beam became slightly distorteddue to different modes beating (image (c)). Near $1557.128 \mathrm{~nm}$ (image (d)), the output beam started to lose power because of efficient light coupling into the cavity. At this strong resonance point, the TLS power was increased in order to have more light out of the microtaper for adequate image processing. The appearance of $\mathrm{LP}_{11}$ mode became much more significant past $1557.128 \mathrm{~nm}$ and tuning the wavelength further causes the LP11 mode to become predominant (images (e) - (h)). Modal transformation from $\mathrm{LP}_{01}$ to $\mathrm{LP}_{11}$ was clearly observed at the 


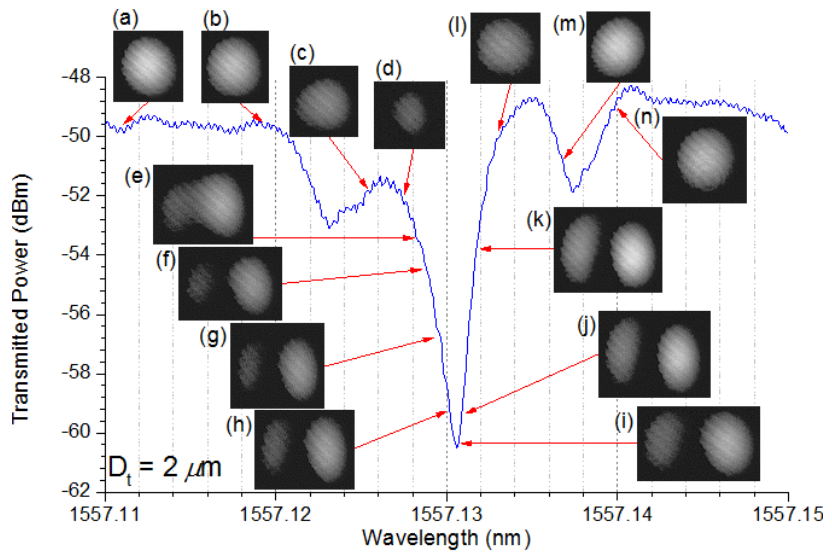

Fig. 8 Near-field images from terminated microtaper fiber of $D_{t}=2$ $\mu \mathrm{m}$ across a MBR WGM.

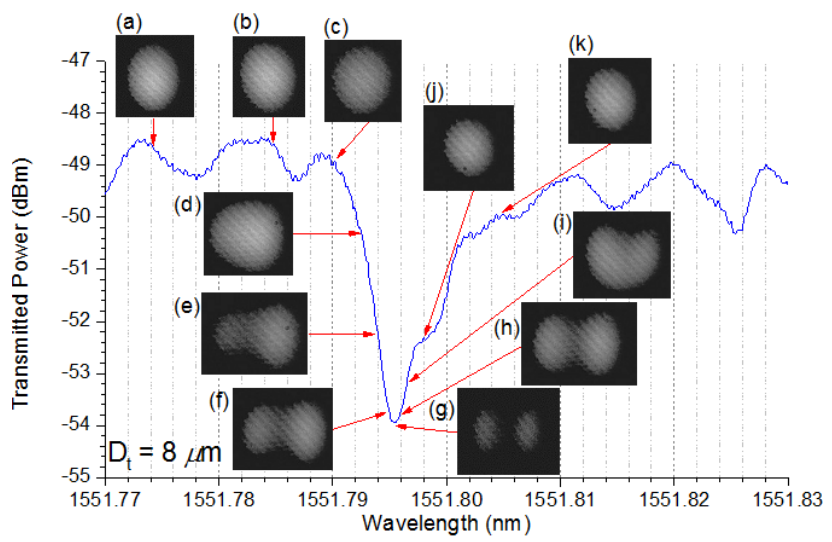

Fig. 9 Near-field images from terminated microtaper fiber of $D_{t}=8$ $\mu \mathrm{m}$ across a MBR WGM.

resonance transmission dip, which was near $1557.131 \mathrm{~nm}$ (image (i)). Again, away from the resonance (past $1557.133 \mathrm{~nm}$ ), only fundamental LP 01 modes were observed (images (l) - (n)). With a few exceptions, most of the other strong WGM resonance checked showed noticeable $\mathrm{LP}_{11}$ mode excitation at their resonance wavelengths. Exciting the MBR with microtaper of $D_{\mathrm{t}}=8 \mu \mathrm{m}$ showed only a small fraction of the WGM resonances to result in a clear $\mathrm{LP}_{01}$ to $\mathrm{LP}_{11}$ modal transformation. Most of the stronger resonances only showed a slight distortion on the output beam image, indicating very small mode transformation. A large number of the resonances checked did not show any noticeable $\mathrm{LP}_{11}$ excitation. Nevertheless, as shown in Fig. 9, the few resonances which did show clear modal transformation follow the same trend as with the previous microtaper with smaller waist diameter. It had been previously reported that coupling 'ideality' from a microresonator back to the desired mode of microtaper fibers would decrease as $D_{t}$ increases [28]. In our study, however, strong $\mathrm{LP}_{01}$ to $\mathrm{LP}_{11}$ modal transformations were observed more frequently on the smaller waist diameter microtaper fibers. Despite the fact that larger waist diameter microtaper fibers support much larger number of modes, the observed modal transformation was significantly smaller and less frequent. The conclusion in [28] was based entirely on microtaper fiber modality arguments. However, mode excitation depends on evanescent field overlaps and phase-matching, which both decrease as the microtaper waist diameter increases. This implies that modal phase-matching, rather than the number of supported modes defines the degree of modal transformation in an evanescently coupled microresonator. As shown in Fig. 10, in the case of $D_{\mathrm{t}}=2 \mu \mathrm{m}$ the effective indices of $\mathrm{LP}_{01}$ and

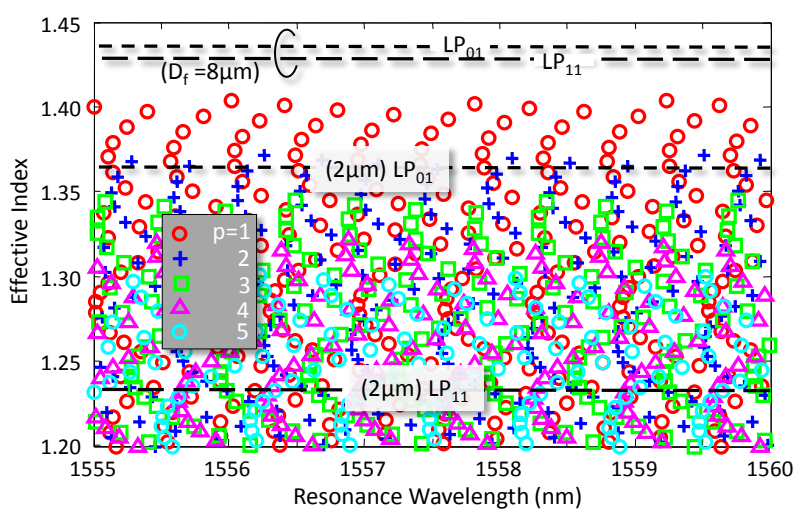

Fig. 10 Effective index of the microtaper $\mathrm{LP}_{01} \& \mathrm{LP}_{11}$ mode $\left(D_{t}=2 \&\right.$ $8 \mu \mathrm{m})$ and MBR WGMs of different radial mode orders $(p=1,2,3,4$, 5), as a function of the resonant wavelength.

$\mathrm{LP}_{11}$ lie well within the effective index range of WGM of different overlapping radial orders, making the $\mathrm{LP}_{01} \rightarrow \mathrm{WGM}_{m p q} \rightarrow \mathrm{LP}_{01}$ and $\mathrm{LP}_{01} \rightarrow \mathrm{WGM}_{m p q} \rightarrow \mathrm{LP}_{11}$ coupling feasible. However, due to its larger evanescent field extension into the MBR, the $\mathrm{LP}_{11}$ mode shows stronger overlap with the $\mathrm{WGM}_{m p q}$ and the $\mathrm{LP}_{01} \rightarrow \mathrm{WGM}_{m p q} \rightarrow \mathrm{LP}_{11}$ transformation is dominant. Similar preferential excitation of $\mathrm{LP}_{11}$ mode has been observed in other coupled optical systems [29]. In the case of $D_{\mathrm{t}}=8 \mu \mathrm{m}$, on the other hand, the effective indices of both $\mathrm{LP}_{01}$ and $\mathrm{LP}_{11}$ are closely together and highly mismatched with the radial order $p$ $=1$ family of WGMs and, therefore, the $\mathrm{LP}_{01} \rightarrow \mathrm{WGM}_{\mathrm{mpq}} \rightarrow \mathrm{LP}_{11}$ mode transformation is not favored.

Finally, we should stress that in the case of full tapered excitation fiber and resonant $\mathrm{LP}_{01} \rightarrow \mathrm{WGM}_{m p q} \rightarrow \mathrm{LP}_{11}$ (or other higher order mode) mode transformation, the $\mathrm{LP}_{11}$ (or higher order) mode will be gradually turn into a cladding mode in the up-taper region of the excitation fiber and be lost. In this case, the mode transformation and subsequent loss will appear as an apparent increased transmission dip and stronger resonance. This effect should be considered when the size of the experimentally obtained transmission dip and resonance wavelength are used to extract information about the MR parameters, such as losses or coupling strength.

\section{FDTD modal simulations}

In order to get additional physical insight, the modal transformation on a waveguide as it excites a WGM microresonator was simulated by utilizing a finite difference time domain (FDTD) software package [30]. The simulation was performed in a 2-D environment with smaller component dimensions relative to the experimental work in order to minimize the required memory size and the run time. The waveguide under study has a $0.7 \mu \mathrm{m}$-wide core with length of $16 \mu \mathrm{m}$. The waveguide was in contact with a microdisc resonator of radius $7 \mu \mathrm{m}$. The waveguide and microdisc refractive index was 1.5 and the background refractive index value was set to 1.0. In order to avoid any back-reflections from the computed windows, perfectly-matched layers were set on the boundaries. The waveguide was two-moded around $\lambda=$ $1 \mu \mathrm{m}$. A TM-fundamental mode pulse was launched from one end of the waveguide with a power monitor placed at the other. The pulse sent into the waveguide core was of Gaussian-shape and centered at $1 \mu \mathrm{m}$ wavelength to calculate the transmission spectrum of the coupled waveguide/microdisc structure. Fig. 11 examines a specific whisperinggallery resonance of the microdisc. The insets show the magnetic $\mathbf{H}$-field intensity patterns across the waveguide near the outputend. Away from 


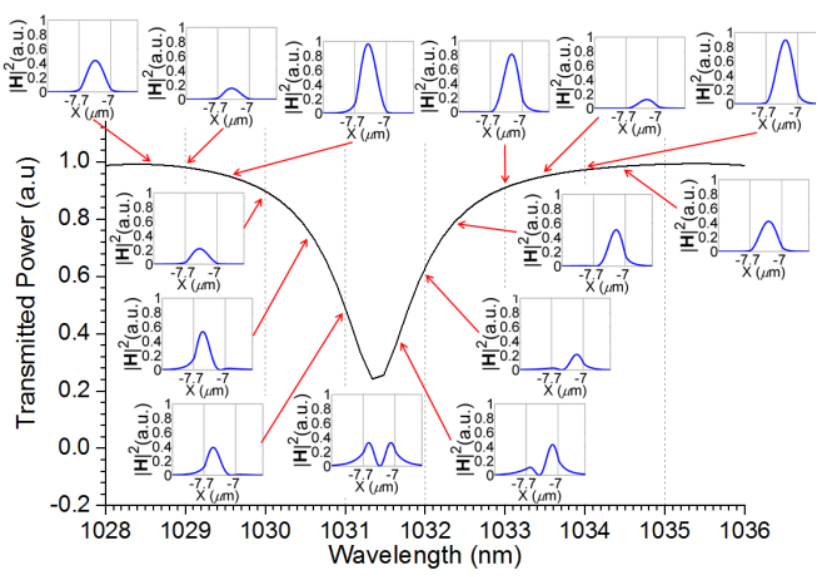

Fig. 11 FDTD simulation $\mathbf{H}$-field intensity patterns acrossa microdisc WGM near the waveguide output end.

resonance (wavelength range $1028.5-1030.0 \mathrm{~nm}$ ), high portion of the launched power remains in the waveguide fundamental $\mathrm{TM}_{0}$ mode. Getting closer to the WGM resonance $(\lambda>1030.5 \mathrm{~nm})$ the output intensity patterns started showing excitations of the waveguide's $\mathrm{TM}_{1}$ mode. At the precise microdisc resonance wavelength of $1031.338 \mathrm{~nm}$, the output $\mathbf{H}$-field intensity pattern shows clear $\mathrm{TM}_{1}$ mode transformation. For longer wavelengths, the $\mathbf{H}$-field patterns consist of two modes travelling through the waveguide. For wavelengths $\lambda>$ $1033.5 \mathrm{~nm}$, only TMo modes were observed at the output of the waveguide. The FDTD simulation performed on a microdisc resonator shows similar trends of energy back-transfer to the waveguide as observed in the MBR experiment. Fig. 12 plots the steady-state $\mathbf{H}$-field distribution within the waveguide and the WGM microdisc resonator at (a) off-resonance wavelength of $1028.500 \mathrm{~nm}$ and (b) on-resonance wavelength of $1031.388 \mathrm{~nm}$. A clear mode transformation is observed when power is coupled from the resonator back into the coupled waveguide at resonance wavelength.

\section{Summary}

In this paper, we have studied in a systematic way, both experimentally and theoretically the effect of the microtaper fiber diameter $\left(D_{t}\right)$ on the MBR transmission spectrum characteristics. Progressively cleaner and simpler spectra were obtained when $D_{t}$ was increased from $2 \mu \mathrm{m}$ to 10 $\mu \mathrm{m}$. Microtaper waist diameters were also shown to have little influence on the loaded $Q$-factor value of the MBR. The measured $Q$-factor with microtaper fiber of different $D_{t}$ were observed to reach values $>10^{6}$ in all cases. Utilization of microtaper fiber with relatively larger waist diameters ( $>6 \mu \mathrm{m}$ ) was observed to increase the transmitted power and generate much simpler spectra, due to excitation of lower-radial-order MBR WGMs, albeit of reduced strength. These features could be beneficial as indication markers when MBRs are intended for sensing purposes [26]. The transmitted power can also be increased in the case of thin microtapers, when the gap between taper and microresonator is accurately controlled [31]. We have also calculated the effect of microtaper diameter on the MBR transmission spectra, showing a good agreement with the experimental results.

Mode transformation as a result of light coupling from MBR back to the microtaper fiber is also studied in detail. It was observed that conversion of the $\mathrm{LP}_{01}$ microtaper fiber mode into $\mathrm{LP}_{11}$ takes place in a number of strong transmission resonances in the case of $2 \mu \mathrm{m}$ taper waist diameters. Despite the much larger modality, and contrary to initial expectations [28], modal transformation was observed to be not as frequent in the case of microtaper with $8 \mu \mathrm{m}$ waist diameter. In this case only a small fraction of the observed resonances showed clear
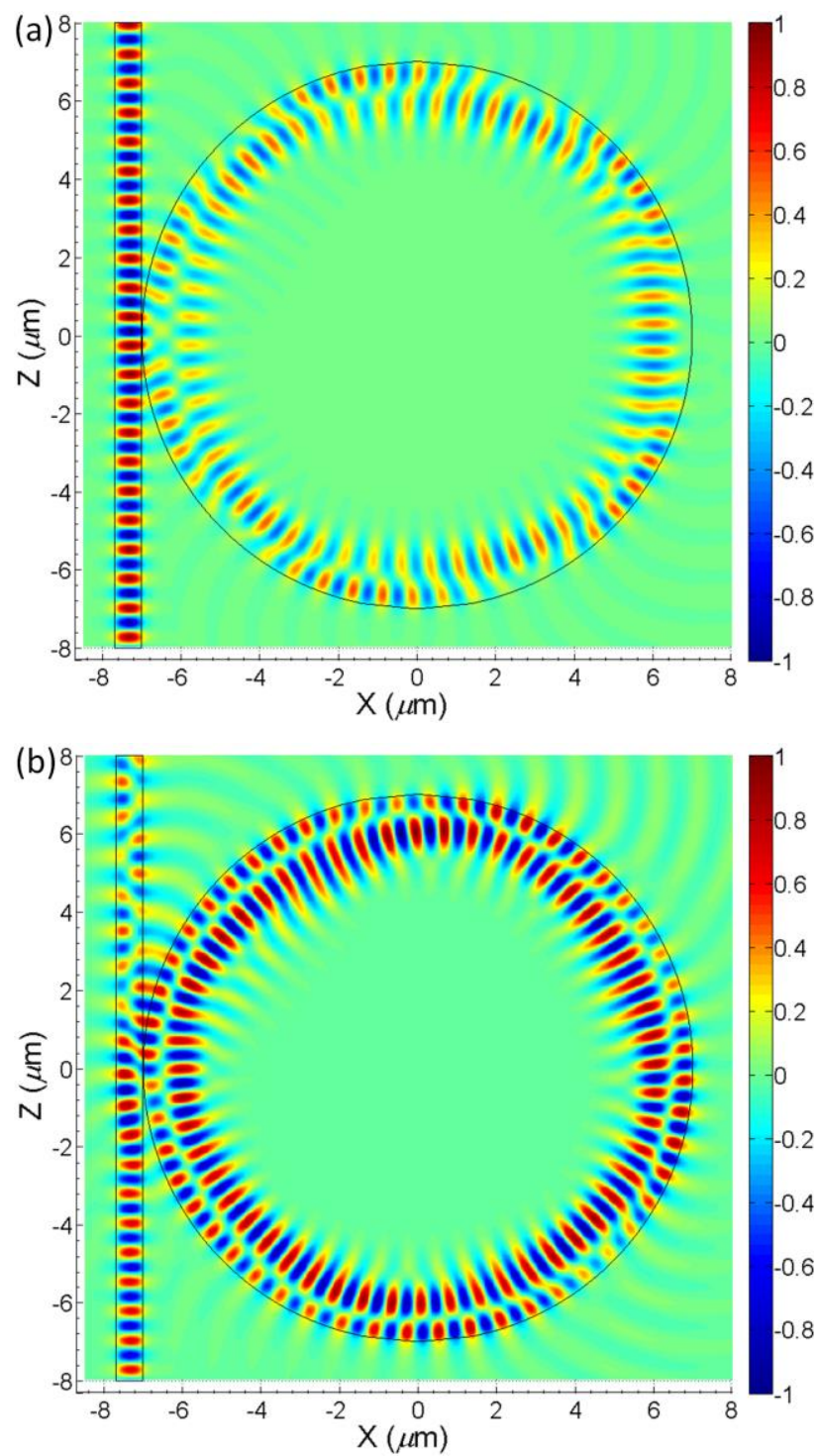

Fig. 12 FDTD steady-state $\mathbf{H}$-field distribution within waveguide and WGM microdisc at (a) off-resonance wavelength of 1028.500 $\mathrm{nm}$ and (b) on-resonance wavelength of $1031.388 \mathrm{~nm}$.

modal transformation from a lower order to a higher order. Using calculations based on a newly developed theoretical model, we demonstrated that modal phase-matching between WGMs and microtaper LP modes, rather than the number of supported LP modes defines the degree of modal transformation and coupling "ideality" in an evanescently coupled microresonator. Hence there is no major concern of increased coupling losses from lower order to higher order modes on MBRs with the utilization of microtaper fibers of larger $D_{t}$. FDTD simulation on a microdisc resonator provides extra physical insightand shows similar energy back-transfer trend to the waveguide as its $\mathbf{H}$-field strengths were scanned across a WGM resonance.

\section{References}

1. K. J. Vahala, "Optical microcavities," Nature 424, 839 (2003).

2. V. S. Ilchenko and A. B. Matsko, "Optical Resonators with WhisperingGallery Modes-Part II: Applications," IEEE Journal of Selected Topics in Quantum Electronics 12, 15 (2006). 
3. M. L. Gorodetsky, A. A. Savchenkov, and V. S. Ilchenko, "Ultimate Q of optical microsphere resonators," Optics Letters 12, 182 (2000).

4. D. K. Armani, T. J. Kippenberg, S. M. Spillane, and K. J. Vahala, "Ultra-high-Q toroid microcavity on a chip," Nature 421, 925 (2003).

5. B. E. Little, J. S. Foresi, G. Steinmeyer, E. R. Thoen, S. T. Chu, H. A. Haus, E. P. Ippen, L. C. Kimerling, and W. Greene, "Ultra-compact Si-SiO2 microring resonator optical channel dropping filters," IEEE Photonics Technology Letters 10, 549 (1998).

6. T. A. Birks, J. C. Knight and T. E. Dimmick, "High-resolution measurement of the fiber diameter variations using whispering gallery modes and no optical alignment," IEEE Photonics Technology Letters 12, 182 (2000).

7. V. S. Ilchenko, M. L. Gorodetsky, X. S. Yao, and L. Maleki, “Microtorus: a highfinesse microcavity with whispering-gallery modes," Optics Letters 26, 256 (2001).

8. M. Sumetsky, Y. Dulashko, and R. S. Windeler, "Optical microbubble resonator," Optics Letters 35, 898 (2010).

9. M. Sumetsky, "Whispering-gallery-bottle-microcavities: the three dimensional etalon," Optics Letters 29, 8 (2004).

10. G. S. Murugan, J. S. Wilkinson, and M. N. Zervas, "Selective excitation of whispering gallery modes in a novel bottle microresonator," Optics Express 17, 11916 (2009).

11. G. S. Murugan, M. N. Petrovich, Y. Jung, J. S. Wilkinson, and M. N. Zervas, "Hollow-bottle optical microresonators," Optics Express 19, 20773 (2011).

12. G. S. Murugan, J. S. Wilkinson, and M. N. Zervas, "Optical excitation and probing of whispering gallery modes in bottle microresonators: potential for all-fiber add-drop filters," Optics Letters 35, 1893 (2010).

13. M. Sumetsky and J. M. Fini, "Surface nanoscale axial photonics," Optics Express 19, 26470 (2011).

14. M. Ding, G. S. Murugan, G. Brambilla, and M. N. Zervas, "Whispering gallery mode selection in optical bottle microresonators," Applied Physics Letters 100, 081108 (2012).

15. V. B. Braginsky, M. L. Gorodetsky, and V. S. Ilchenko, "Quality-factor and nonlinear properties of optical whispering-gallery modes," Physics Letters A 137, 393 (1989).

16. M. L. Gorodetsky and V. S. Ilchenko, "Optical microsphere resonators: optimal coupling to high-Q whispering gallery modes," Journal of Optical Society America B 16, 147 (1999).

17. V. S. Ilchenko, X. S. Yao, and L. Maleki, "Pigtailing the high-Q microsphere cavity: a simple fiber coupler for optical whispering-gallery modes," Optics Letters 24, 723 (1999).

18. L. Arques, A. Carrascosa, V. Zamora, A. Díez, J. L. Cruz, and M. V. Andrés, "Excitation and interrogation of whispering-gallery modes in optical microresonators using a single fused-tapered fiber tip," Optics Letters 36, 3452 (2011).

19. J. C. Knight, G. Cheung, F. Jacques, and T. A. Birks, "Phase-matched excitation of whispering-gallery-mode resonances by a fiber taper," Optics Letters 22, 1129 (1997).

20. O. Svitelskiy, Y. Li, A. Darafsheh, M. Sumetsky, D. Carnegie, E. Rafailov, and V.S. Astratov, "Fiber coupling to BaTiO3 glass microspheres in an aqueous environment," Optics Letters 36, 2862 (2011).

21. B. E. Little, J. P. Laine and H. A. Haus, "Analytic theory of coupling from tapered fibers and half-blocks into microsphere resonators," Journal of Lightwave Technology 17, 704 (1999).

22. Y. Panitchob, G.Senthil Murugan, M. N. Zervas, P. Horak, S. Berneschi, S. Pelli, G.Nunzi Conti, and J. S. Wilkinson, "Whispering gallery mode spectra of channel waveguide coupled microspheres," Optics Express 16, 11076 (2008).

23. M. N. Zervas, "Transmission resonance modelling in microbottle resonators,"'" under preparation.

24. A. Yariv, "Universal relations for coupling of optical power between microresonators and dielectric waveguides," Electronics Letters 36, 321 (2000).

25. G. Lin, B. Qian, F. Orucevic, Y. Candela, J.-B. Jager, Z. Cai, V. LefèvreSeguin, and J. Hare, “Excitation mapping of whispering gallery modes in silica microcavities”, Optics Letters 35, 583 (2010).
26. M. N. Mohd Nasir, G. S. Murugan, and M. N. Zervas, "Broadly Tunable Solid Microbottle Resonator," submitted to 2016 IEEE Photonics Conference (IPC), Hawaii USA.

27. J. Villatoro, D. Monzón-Hernández, and E. Mejía, "Fabrication and modeling of uniform-waist single-mode tapered optical fiber sensors," Applied Optics 42, 2278 (2003).

28. S. M. Spillane, T. J. Kippenberg, O. J. Painter, and K. J. Vahala, "Ideality in a Fiber-Taper-Coupled Microresonator System for Application to Cavity Quantum Electrodynamics," Physical Review Letters 91, 043902 (2003).

29. S. Barcelos, M. N. Zervas and P. S. J. Russell, "Selective excitation of fibermodes using surface plasmons," IEEE Photonics Technology Letters 9, 1051 (1995).

30. RSoft Design Group, Rsoft Inc., FullWAVE 6.0 (2008).

31. M. Cai, O. Painter, and K. J. Vahala, "Observation of Critical Coupling in a Fiber Taper to a Silica-Microsphere Whispering-Gallery Mode System," Physical Review Letters 85, 74 (2000). 